\title{
REFERENCES
}

1. H. Cartan and S. Eilenberg, Homological algebra, Princeton University Press, 1956.

2. I. Kaplansky, Modules over Dedekind rings and valuation rings, Trans. Amer. Math. Soc. vol. 72 (1952) pp. 327-340.

3. E. Matlis, Injective modules over Noetherian rings, Pacific J. Math. vol. 8 (1958) pp. 511-528.

NORTHWESTERN UNIVERSITY

\section{A CHARACTERIZATION OF ALGEBRAIC NUMBER FIELDS WITH CLASS NUMBER TWO ${ }^{1}$}

\section{CARLITZ}

Let $Z=R(\theta)$ denote an algebraic number field over the rationals with class number $h$. It is familiar that $h=1$ if and only if unique factorization into prime holds for the integers of $Z$. For fields with $h \leqq 2$ we have the following criterion.

THEOREM. The algebraic number field $Z$ has class number $\leqq 2$ if and only if for every nonzero integer $\alpha \in Z$ the number of primes $\pi_{j}$ in every factorization

$$
\alpha=\pi_{1} \pi_{2} \cdots \pi_{k}
$$

depends only on $\alpha$.

Suppose first that $h=2$ and consider the factorization into prime ideals

$$
(\alpha)=\mathfrak{p}_{1} \cdots \mathfrak{p}_{s} \mathfrak{r}_{1} \cdots \mathfrak{r}_{t},
$$

where the $\mathfrak{p}_{j}$ are principal ideals while the $\mathfrak{r}_{j}$ are not. Then

$$
p_{j}=\left(\pi_{j}\right) \quad(j=1, \cdots, s) .
$$

Since $h=2$, it follows that

$$
\mathfrak{r}_{i} \mathfrak{r}_{j}=\left(\rho_{i j}\right) \quad(i, j=1, \cdots, t) ;
$$

moreover $t$ must be even, $=2 u$, say. Thus every factorization into primes implied ". by (2), for example

Received by the editors August 3, 1959.

1 Research sponsored by National Science Foundation grant NSF G-9425. 


$$
\alpha=\epsilon \pi_{1} \cdots \pi_{s} \rho_{12} \cdots \rho_{t-1, t},
$$

where $\epsilon$ is a unit, will contain exactly $s+u$ primes.

We now show that when $h>2$, there occur factorizations (1) with different values of $k$. The proof makes use of the fact that every class of ideals contains at least one prime ideal. (For proof of a much stronger result see [1]).

Assume first the existence of a class $A$ of period $m>2$. Let $\mathfrak{p}$ be a prime ideal in $A$ and $\mathfrak{p}^{\prime}$ a prime ideal in $A^{-1}$. Then we have

$$
\mathfrak{p}^{m}=(\pi), \quad \mathfrak{p}^{\prime m}=(\pi)^{\prime}, \quad \mathfrak{p p}^{\prime}=\left(\pi_{1}\right),
$$

and it is easily verified that $\pi, \pi^{\prime}, \pi_{1}$ are primes. Clearly (3) implies

$$
\pi_{1}^{m}=\epsilon \pi \pi^{\prime},
$$

where $\epsilon$ is a unit.

In the next place assume the existence of two classes $A_{1}, A_{2}$ each of period 2 such that $A_{3}=A_{1} A_{2}$ is not principal. Choose prime ideals $\mathfrak{p}_{j} \in A_{j}(j=1,2,3)$. Then we have

$$
p_{j}^{2}=\left(\pi_{j}\right)(j=1,2,3), \quad \mathfrak{p}_{1} p_{2} p_{3}=(\pi),
$$

and again it is easily verified that $\pi_{1}, \pi_{2}, \pi_{3}, \pi$ are all primes. From (5) we get

$$
\pi^{2}=\pi_{1} \pi_{2} \pi_{3}
$$

Using (5) and (6) it is evident that when $h>2$, the number of primes $k$ in (1) is not independent of the factorization.

Since the case $h=1$ requires no further discussion, this completes the proof of the theorem.

\section{REFERENCE}

1. E. Hecke, Über die L-Funktionen und den Dirichletschen Primzahlsatz für einen beliebigen Zahlkörper, Nachr. Akad. Wiss. Göttingen. Math.-Phys. Kl. IIa (1917) pp. 299-318.

DUKE UNIVERSITY 